\title{
Cirurgia em peixes
}

Fish surgery

\section{Cirugía de pescado}

Recebido: 02/11/2020 | Revisado: 07/11/2020 | Aceito: 10/11/2020 | Publicado: 13/11/2020

\section{Jaqueline Nunes Nieri Moreira da Rocha}

ORCID: https://orcid.org/0000-0002-9139-4062

Centro Universitário Serra dos Órgãos, Brasil

E-mail: jackienr@terra.com.br

Antonia Cleia Bastos

ORCID: https://orcid.org/0000-0002-0932-5835

Centro Universitário Serra dos Órgãos, Brasil

E-mail: antoniacleia@yahoo.com.br

Mayra Meneguelli

ORCID: https://orcid.org/0000-0002-6369-958X

Universidade Federal de Rondônia, Brasil

E-mail: mayrameneguelli@gamil.com

Felipe Mateus Berndt

ORCID: https://orcid.org/0000-0001-8211-5095

Universidade Federal de Rondônia, Brasil

E-mail: felipeberndt@ hotmail.com

Talita Oliveira Mendonça

ORCID: https://orcid.org/0000-0001-6739-7071

Universidade Estadual Paulista Júlio de Mesquita Filho, Brasil

E-mail: talita.medvet18@gmail.com

Dayane da Silva Zanini

ORCID: https://orcid.org/0000-0002-5457-5016

Universidade Federal de Rondônia, Brasil

E-mail: dayanezanini.medvet@gmail.com

Igor Mansur Muniz

ORCID: https://orcid.org/0000-0003-0863-6647

Universidade Federal de Rondônia, Brasil

E-mail: igor.mansur@unir.br 


\title{
Resumo
}

A aquicultura e o mercado de peixes ornamentais vêm conquistando um papel de destaque no cenário do agronegócio no Brasil e no mundo, fazendo com que cresça uma busca por aprimoramento profissional nesta área, por parte de médicos veterinários. Com isso, este trabalho buscou, através de uma investigação por meio de revisão de literatura, explorar o tema de cirurgia em peixes, com o objetivo de promover um levantamento dos principais procedimentos cirúrgicos executados nestes animais. Justifica-se a importância deste estudo pela atual necessidade da realização de procedimentos cirúrgicos em certos peixes, devido ao seu alto valor econômico e sentimental, além da raridade de algumas espécies ornamentais e exóticas. Apesar da pouca disponibilidade de material para pesquisa, foi possível com este estudo reunir informações importantes acerca dos procedimentos de cirurgias em peixes, ficando constatado ser esta uma área da medicina veterinária que ainda necessita de expansão, através de pesquisas, melhoria de técnicas e publicações, pois ainda há muitas lacunas a serem preenchidas.

Palavras-chave: Cirurgia; Anestesia; Peixes.

\begin{abstract}
The aquaculture and ornamental fish market is gaining a prominent role in the scenario of agribusiness in Brazil and worldwide, causing it to grow a search for professional development in this area by veterinarians. Thus, this study sought, through an investigation by means of literature review, exploring the theme of surgery in fish, with the goal of promoting a survey of major surgical procedures performed in these animals. Justifies the importance of this study for the current need for surgical procedures in certain fish due to its high economic value and sentimental, and the rarity of some ornamentals and exotic. Despite the limited availability of research material, it was possible in this study to gather important information about the procedures of surgeries in fish, this being found to be an area of veterinary medicine that still needs expansion, through research, publications and technical improvement, because there are still many gaps to be filled.
\end{abstract}

Keywords: Surgery; Anesthesia; Pisces.

\section{Resumen}

La acuicultura y el mercado de peces ornamentales han ido ganando un papel destacado en el escenario de la agroindustria en Brasil y en el mundo, provocando una búsqueda de superación profesional en esta área, por parte de los veterinarios. Con ello, este trabajo buscó, 
a través de una investigación a través de la revisión de la literatura, explorar el tema de la cirugía en peces, con el objetivo de promover un relevamiento de los principales procedimientos quirúrgicos realizados en estos animales. La importancia de este estudio se justifica por la necesidad actual de realizar procedimientos quirúrgicos en determinados peces, debido a su alto valor económico y sentimental, además de la rareza de algunas especies ornamentales y exóticas. A pesar de la limitada disponibilidad de material para la investigación, con este estudio fue posible recabar información importante sobre los procedimientos de cirugía en peces, que resultó ser un área de la medicina veterinaria que aún necesita expansión, a través de la investigación, mejora de técnicas y publicaciones. ya que aún quedan muchas lagunas por llenar.

Palabras clave: Cirugía; Anestesia; Piscis.

\section{Introdução}

O setor de peixes ornamentais vem ganhando um papel significativo no comércio mundial, na pesca, aquicultura e no desenvolvimento socioeconômico, sendo que a maior parte da produção de aquicultura de peixe ornamental se concentra em espécies de água doce (Chambel, et al., 2015).

Diante desse cenário também se iniciou a busca por aprimoramento profissional, por parte de alguns médicos veterinários, em ictiopatologia, parasitologia, hematologia, microbiologia, diagnósticos laboratoriais, além de orientação na utilização das principais ferramentas de diagnóstico em peixes. Além destas especializações, Harms \& Lewbart (2000) afirmam que a cirurgia em peixes atualmente é viável e prática para aplicações clínicas e de pesquisa.

Sendo assim, este trabalho buscou explorar o tema de cirurgia em peixes, com o objetivo de promover um levantamento dos principais procedimentos cirúrgicos executados nestes animais. Justifica-se a importância deste estudo pela atual necessidade da realização de procedimentos cirúrgicos em certos peixes, devido ao seu alto valor econômico e sentimental, além da raridade de algumas espécies ornamentais e exóticas.

\section{Metodologia}

Com o objetivo de promover um levantamento dos principais procedimentos cirúrgicos executados em peixes ornamentais, utilizou-se como suporte as literaturas Koche 
(2011) e Pereira et al. (2018) para orientar a produção dessa revisão de literatura.

A coleta de dados bibliográficos foi realizada nos anos de 2012 e atualizadas em 2019 por meio da base de dados Pubmed, Google Acadêmico e Scielo.

Foram empregados alguns critérios de inclusão e exclusão para a seleção de artigos. Dentre eles os critérios de inclusão foram: (1) Peixes ornamentais; (2) Anestesia em peixes; (3) Cirurgia em peixes.

\section{Resultados e Discussão}

A pesquisa bibliográfica identificou 34 artigos nas três bases de dados utilizadas, e pelo software Endnote web não foram encontrados artigos duplicados.

\subsection{Aspectos anatômicos de peixes}

Os peixes são classificados como vertebrados, estando distribuídos nas classes Condrictes e Osteíctes. (Maranho, 2007), pertencentes à superclasse Pisces, subfilo Vertebrata, filo Chordata (Pereira, 2015).

A anatomia externa do corpo do peixe é composta por boca, narina, olho, opérculo, nadadeiras (peitoral, pélvica, dorsal, caudal e anal), escamas e ânus, e internamente, brânquias, coração, faringe, fígado, estômago, intestino, gônadas, bexiga urinária, bexiga natatória, rim, coluna vertebral, medula espinhal e cérebro (Paske \& Lanzendorf, 2017).

\subsection{Procedimentos cirúrgicos em peixes}

Assim como os mamíferos terrestres, os peixes também passam por procedimentos cirúrgicos, como implantação de telemetria; remoção, avaliação ou biópsia de órgãos internos e externos; e remoção de materiais estranhos (Cannizzo, et al., 2016).

Além de processos patológicos, há também outros motivos que levam a intervenção cirúrgica. A implantação intraperitoneal cirúrgica de transmissores de ultrassom, transmissores de rádio e transponders passivos integrados tornou-se uma técnica comum para acompanhamento da utilização movimento e habitat de um indivíduo. Os recentes avanços tecnológicos na miniaturização de componentes eletrônicos têm facilitado o uso de transmissores para monitorar peixes cada vez menores (Cooke, et al., 2003; Wagner \& Cooke, 2005). 


\subsection{Principais procedimentos cirúrgicos em peixes}

Pires (2009) cita como motivos de intervenções mais comuns: Acumulo de conteúdo caseoso; Parasitas; Torções; Rompimentos; Hiper ou hipoinflação da bexiga natatória; Deslocamentos; Neoplasias.

Reid \& Backman (1988) relatam a excisão de um tumor de um peixe de $25 \mathrm{~cm}$, Cyclosoma labiatum da ordem Cichlidae. O tumor se localizava no aspecto caudoventral da nadadeira dorsal e tinha aproximadamente oito $\mathrm{cm} 3$, com hiperemia em torno da sua periferia. Foi realizada a anestesia e em seguida a cirurgia, que foi executada na água, em decúbito ventral, porém com o local cirúrgico mantido fora da água. A massa foi removida utilizando técnicas padrão de excisão de tumores. Um cautério térmico foi utilizado para selar o local de excisão e evitar a perda de eletrólitos. O peixe evoluiu bem em um tanque separado oxigenado e foi então transferido para um tanque de convalescença contendo $0,5 \%$ de solução de $\mathrm{NaCl}$ (cloreto de sódio), durante um período de uma semana. O tumor era um fibroma benigno com um foco inflamatório.

Um procedimento citado por Pires (2009) é a resolução de hiperinflação da bexiga natatória. $O$ pesquisador explica que deve-se pesar o animal e em seguida usar o que se chama de princípio de Arquimedes, onde se coloca o peixe já pesado em um recipiente com água e observa-se o quanto de volume de água ele irá deslocar. Por exemplo, um peixe de $47 \mathrm{~g}$ deve deslocar $47 \mathrm{ml}$ de água. Se ele deslocar $50 \mathrm{ml}$ terá deslocado $3 \mathrm{ml}$ a mais que seu peso, onde 3 $\mathrm{ml}$ equivalem a $3 \mathrm{~cm} 3$, sendo este o volume de bexiga natatória que poderá ser removido na cirurgia. Esta cirurgia é feita mediante uma celiotomia.

A respeito da implantação de transmissores de telemetria em peixes, o procedimento cirúrgico básico envolve a inserção no peixe de um transmissor através de uma incisão para dentro da cavidade peritoneal, que é posteriormente selada. A maioria dos métodos cirúrgicos utilizados permanece inalterada, e testes empíricos pouco têm sido feitos. Apesar de telemetria ter a capacidade de fornecer uma riqueza de informações ecológicas e fisiológicas, se a saúde dos peixes estiver comprometida com o processo de implantação do transmissor, os dados assim adquiridos serão suspeitos. O comprometimento fisiológico pode levar a mudanças de comportamento dos peixes, ou seja, o mesmo dado que o estudo está tentando medir (Wagner \& Cooke, 2005; Wagner, et al., 2011).

Por exemplo, o procedimento descrito pelo instituto de pesquisas americano Pfleger Institute of Environmental Research (Pier, 2012), relata um procedimento de colocação de transmissor em peixe feito sem uso de anestesia. A descrição se resume no seguinte: cada 
peixe é capturado com armadilhas ou por linha e anzol e depois trazido a bordo do barco, então o peixe é contido em uma placa acolchoada cirúrgica, com água do mar fresca irrigando suas guelras, sem anestésicos, o transmissor acústico é implantado cirurgicamente na cavidade peritoneal do peixe, a incisão é fechada com suturas estéreis e o peixe é liberado de volta para a área onde ele foi capturado.

Wagner \& Cooke (2005) alertam que, para o desenvolvimento de protocolos adequados para a cirurgia de implantação de transmissores em peixes, é importante compreender que a grande maioria dos estudos que envolvem a implantação cirúrgica destes transmissores é realizada no campo. Estes locais de estudo remotos criam vários desafios para os pesquisadores, incluindo a operação de bateria de bombas de água e luzes, condições climáticas adversas, dificuldades em manter o equipamento estéril e os campos cirúrgicos. Além disso, dado que a água usada durante a cirurgia, e na qual os peixes são subsequentemente colocados, não é estéril, torna-se óbvio que os regulamentos de metodologia para a cirurgia de peixes requerem modificações únicas para que possam ser aplicados pelos investigadores que se destinam atingir.

\subsection{Considerações pré-cirúrgicas}

Brattelid \& Smith (2000) alertam que a remoção do peixe de seu meio natural pode causar danos irreparáveis no tegumento, bem como uma grande variedade de alterações fisiopatológicas do animal. A pele de peixe é altamente sensível e pode ser facilmente danificada por manipulação, tendo em vista que, ao contrário da pele dos mamíferos, a sua camada exterior é constituída por células vivas.

A pele do peixe é facilmente danificada por alcoóis e soluções de paramentação cirúrgicas, e a resposta exacerbada das glândulas produtoras de muco ao dano excede em muito a quaisquer benefícios potenciais resultantes da sua utilização. Portanto, não se usa álcool em peixes (Stoskopf, 1993).

Diluições de iodopovidona têm sido usadas sem prejuízo para algumas espécies, mas pode queimar quimicamente a pele de algumas outras espécies de peixes. O problema de manter um ambiente estéril na cirurgia de peixe é ainda mais complicado pela necessidade de manter a umidade do paciente em todos os momentos e fornecer alta qualidade de água para o animal a respirar (Stoskopf, 1993). Em vista destas dificuldades, Pires (2009) sugere o uso de filme plástico para isolar a área do campo cirúrgico.

Brattelid \& Smith (2000) também consideram que uma mesa de operação padrão plana 
está longe de ser satisfatória, tanto para o peixe como para o cirurgião, esses autores sugerem que um suporte físico para posicionamento cirúrgico ideal deve satisfazer os seguintes critérios: não deve danificar o tegumento e deve ser ajustável para se adaptar ao tamanho e forma do peixe em questão; deve possuir uma superfície lisa, mas reter o peixe na posição necessária; deve ter uma superfície que não absorve a água, deve ser fácil de manter limpo e desinfetado; deve apoiar o peixe ao longo de toda sua extensão; não pode ter bordas afiadas; deve facilitar a irrigação das brânquias com água corrente; deve ser fácil de manusear, não deve reagir com água, desinfetantes ou soluções anestésicas; não deve conduzir calor; não deve perturbar ou interferir com o procedimento experimental; deve ser possível ajeitá-lo à mesa de operação; deve ser adaptável a uma vasta gama de tamanhos de peixe e deve ser relativamente barato.

Estes mesmos autores revelam que inúmeras pesquisas descrevem o uso do suporte de calha em formato de $\mathrm{V}$ ou $\mathrm{U}$, construídos de uma variedade de materiais e com diferentes acabamentos. Os pregos ou parafusos devem ser de aço inoxidável e não podem ser galvanizadas ou construídas de qualquer liga que contenha cobre ou outros metais tóxicos, caso a solução anestésica for do tipo reutilizável. Sendo de madeira, a mesma não deve ser tratada com qualquer produto químico de verniz. A utilização de um pano úmido vai ajudar a manter a pele umedecida e impedir a ruptura nos pontos em que o corpo do animal faz contato com a calha (Brattelid \& Smith, 2000).

Sugere-se o uso de antibióticos pré-operatórios em alguns casos, no intuito de evitar infecções. Ainda não se dispõe de uma avaliação sistemática dos peixes em relação à eficácia da terapia pré-operatória profilática de antibióticos, porém, dependendo da natureza do procedimento cirúrgico, do local onde a cirurgia foi realizada e o grau de possível monitorização pós-operatória, recomenda-se o uso de enrofloxacina de $5-10 \mathrm{mg} / \mathrm{kg}$ IM (intramuscular), ou oxitetraciclina, $10 \mathrm{mg} / \mathrm{kg}$ IM. Se a cirurgia envolver penetração do intestino ou feridas contaminadas, pode-se promover a continuação de antibióticos (Harms, 1998).

\subsection{Considerações anestésicas}

Um anestésico ideal deve induzir a anestesia rapidamente com mínima hiperatividade ou stress. Ela deve ser de fácil administração e deve manter o animal no estado anestésico escolhido. Quando o animal é removido do anestésico, a recuperação deve ser rápida. O anestésico deve ser eficaz em doses baixas e a dose tóxica deve exceder em muito a dose 
eficaz, de modo que exista uma ampla margem de segurança (Coyle; Durborow; Tidwell, 2004).

O procedimento cirúrgico primeiramente precisa passar pela etapa de administração de uma correta anestesia, para eliminar a dor. Embora os peixes possam ser injetados com anestésicos, a grande maioria dos procedimentos anestésicos é realizada por imersão ou através de um tratamento de irrigação estática ou com água corrente. Em ambos os casos, o anestésico deve ser solúvel em água. Em alguns casos, os anestésicos são primeiro dissolvidos num solvente orgânico e em seguida diluídos em água. O peixe, então, é exposto à solução aquosa do composto a uma concentração pré-determinada, por um período de tempo específico. As concentrações são tipicamente calculadas em termos de partes por milhão (ppm), o que é equivalente a miligramas por litro de água $(\mathrm{mg} / \mathrm{L})$ ou gramas por metro cúbico de água $\left(\mathrm{g} / \mathrm{m}^{3}\right)$. O grau de sedação do peixe (Tabela 1) é uma função da concentração do composto e da duração da exposição (Browser, 2001).

Tabela 1. Estágios da anestesia.

\begin{tabular}{cll}
\hline Estágios & Descritor & Resposta comportamental do peixe \\
\hline $\mathbf{0}$ & Normal & $\begin{array}{l}\text { Reativo a estímulos externos; taxa opercular e } \\
\text { tônus muscular normal. }\end{array}$ \\
$\mathbf{1}$ & Sedação leve & Ligeira perda de reatividade a estímulos \\
& & externos; taxa opercular diminuiu \\
& ligeiramente; equilíbrio normal.
\end{tabular}

Fonte: Brown, (1993). 
Research, Society and Development, v. 9, n. 11, e2659119849, 2020

(CC BY 4.0) | ISSN 2525-3409 | DOI: http://dx.doi.org/10.33448/rsd-v9i11.9849

Além dos estágios já citados, Roubach \& Gomes (2001) acrescenta o de Recuperação, que consiste na recuperação do equilíbrio pelo peixe e natação normal.

A anestesia transmitida pela água é a rota mais utilizada de administração de anestésico para peixes. Para o tratamento da água, a droga pode ser trazida para a concentração desejada na própria água que contém o peixe ou o peixe pode ser colocado em um tanque que contém a indução da anestesia. Pequenos procedimentos clínicos podem ser realizados em peixes anestesiados, brevemente removidos da água para as manipulações necessárias, e devolvidos ao tanque de anestesia para estender o procedimento ou serem movidos para a água de recuperação, quando o procedimento é concluído. Porém, cirurgias que envolvam maior tempo fora da água requerem uma distribuição contínua de água com anestesia para as guelras do peixe. Um volume baixo, sem recirculação, em um sistema de alimentação por gravidade que compreende um reservatório de saco de fluido IV (intravenoso), de gotejamento conjunto e cateter de borracha, funciona bem para os peixes pequenos. Sistemas de maior capacidade de recirculação foram descritos usando dois tanques com uma bomba ou um sifão e uma bomba (Lewbart \& Harms, 1999).

Mas apesar de o método mais comum de administração de fármaco anestésico em peixes ser por meio de imersão (que é uma técnica análoga à anestesia de inalação gasosa nos animais terrestres), o uso de agentes anestésicos injetáveis e sedativos (principalmente por via intramuscular, mas também por via intravenosa) é crescente (Neiffer \& Stamper, 2009).

Segundo Pires (2009), o fármaco anestésico a ser utilizado em uma cirurgia de peixe irá depender do animal a ser anestesiado e do procedimento a ser realizado. O fármaco mais utilizado é o MS-222 (tricaina metanossulfonato), porém também são utilizados o Isoflurano, Ketamina, Propofol e Benzocaina.

Já Roubach \& Gomes (2001) citam como anestésicos mais utilizados no Brasil os seguintes: MS-222, Benzocaína, Quinaldina (2-4 metilquinolina) ou Quinaldina sulfato (sulfato de 2-4 metilquinolina), 2-fenoxietanol ou Etileno glicol éter fenil éter, Mentol. A Tabela 2 que se segue esclarece mais detalhes sobre cada um deles. 
Tabela 2. Forma de utilização dos anestésicos mais comumente encontrados no Brasil.

\begin{tabular}{lll}
\hline Anestésico & Forma de apresentação & Preparo da solução e estocagem
\end{tabular}

MS-222 Pó

Benzocaína

Pó

Quinaldina Líquido

2-fenoxietanol Líquido

Mentol

Cristal
Colocado diretamente na água. Por ser muito ácido é aconselhável tamponar a água com bicarbonato de sódio, até se obter um $\mathrm{pH}$ semelhante ao de antes da aplicação do produto. Se preferir, pode-se preparar uma solução mãe (10g de MS-222 em um litro de água destilada). Esta solução é efetiva por três meses se estocada na geladeira. Após aberto, o produto deve ser mantido em geladeira.

Deve ser dissolvido em acetona ou álcool. Normalmente se prepara uma solução mãe de $100 \mathrm{~g}$ de benzocaína em um litro de solvente. Deve-se armazenar em vidro escuro, podendo durar até um ano.

Deve ser dissolvido em acetona ou álcool antes de ser misturado à água $(100 \mathrm{ml}$ do líquido para $200 \mathrm{ml}$ de solvente). Por ser muito ácido é aconselhável tamponar a água com bicarbonato de sódio, até se obter um $\mathrm{pH}$ semelhante ao de antes da aplicação do produto. Deve-se armazenar em vidro escuro.

Colocado diretamente na água. Continua ativo na água por até três dias. Deve ser armazenado no frasco do fabricante.

Deve ser dissolvido em acetona ou álcool. Normalmente se prepara uma solução mãe de $100 \mathrm{~g}$ de mentol em um litro de solvente. Deve ser armazenado em vidro escuro.

Fonte: Roubach \& Gomes, (2001).

Mas além destes já citados, há também o uso do óleo de cravo como anestésico em peixes. Este anestésico, como explica Bizarro (2011), é muito utilizado em peixes para práticas rotineiras (necessárias na piscicultura), como biometria, análise patológica, implante hormonal e principalmente o transporte, tendo em vista que são procedimentos que envolvem manipulação direta dos animais, o que leva ao estresse dos mesmos, o que pode causar perda de desempenho e problemas de sanidade. Assim, o anestésico seria utilizado nestes casos para evitar o estresse, diminuindo a interferência nas funções vitais e fisiológicas dos peixes.

A eficiência e segurança do óleo de cravo para anestesia são descritas na literatura para várias espécies de peixes, tanto para o manejo nas condições de criação intensiva, quanto nas pesquisas em laboratório (Mazzafera, 2003; Pereira-Da-Silva, et al., 2009). 
Muitas vezes, a velocidade em que as drogas anestésicas tornam-se eficazes está relacionada com a área das guelras em relação ao peso corporal, o que pode variar consideravelmente entre as espécies de peixes. Além disso, espécies aquáticas também têm diferentes taxas metabólicas que afetam o ritmo em que os produtos químicos são absorvidos e a anestesia é induzida. Existem também fatores que podem afetar a anestesia dentro de uma determinada espécie. Indivíduos maiores geralmente exigem uma maior concentração de anestésico que os indivíduos menores. Em compensação, também tem sido relatado que peixes maiores e mais ativos num grupo são anestesiados mais rápido do que os menores (Coyle; Durborow; Tidwell, 2004). Muitas drogas, tais como MS-222 e benzocaína são solúveis em gordura e, portanto, em peixes maiores ou fêmeas grávidas, a anestesia pode durar mais tempo e a recuperação pode ser mais lenta, até que a droga seja removida das reservas lipídicas. Além disso, os animais doentes ou debilitados são muito mais suscetíveis ao tratamento anestésico (Chambel, 2015).

\subsection{Suturas}

A agulha para sutura deve ser a menor e menos invasiva, para penetrar facilmente na pele e tecidos subjacentes, deixando menor furo possível e minimizar o dano ao tecido. Uma agulha com uma curvatura de três oitavos de circunferência é geralmente utilizada para suturar a pele porque exige apenas ligeiro movimento do pulso. As agulhas com um desenho circular também podem ser usadas, mas requerem mais movimento de mão, o que pode causar desconforto e fadiga mais rapidamente para os cirurgiões (Wagner, et al., 2011).

Muitos tipos de sutura já foram utilizados em peixes. Porém suturas sintéticas não são absorvidas nestes animais. Geralmente recomenda-se usar monofilamento na pele para diminuir o risco de infecção na ferida cirúrgica. Outros fatores em relação à sutura se referem aos tecidos e tempo de cicatrização. Resultados preliminares de um estudo em carpa indicam que fios de sutura como monofilamento ou fibra sintética e poligliconato são ligeiramente superiores à sutura trançada (Hurty, et al., 2002).

É importante não atar as suturas com muita força, pois isto pode causar estrangulamento do tecido, levando a necrose isquêmica, bem como a ausência de aposição das camadas de tecido (Wagner, et al., 2011).

Porém Harms (1998) diz que outros tipos de sutura também podem ser utilizados, como o padrão de sutura contínua, tendo sido efetuado com sucesso em vários estudos. Padrões contínuos de sutura têm a vantagem de minimizar área de superfície disponível para 
colonização microbiana, além de redução do tempo de cirurgia, mas podem ser mais propensas ao afrouxamento se uma tensão adequada não for mantida através da linha inteira e se os nós nas extremidades não estiverem seguros. Portanto, os padrões suspensos podem ser mais apropriados para os cirurgiões inexperientes. O ideal é remover as suturas cutâneas quando a incisão estiver curada, o que geralmente ocorre em 2 a 3 semanas. Normalmente a remoção de suturas elimina uma fonte de inflamação e pode acelerar as fases finais da cicatrização da incisão.

Ao escolher o material de sutura para fechar a incisão, uma prática geralmente aceita é a de utilizar a sutura de menor diâmetro, com tensão adequada. No entanto, sugere-se que antes de se realizar a cirurgia, o peixe seja testado com a sutura proposta para assegurar que o material não seja pequeno demais, pois iria indevidamente cortar a pele no local da incisão. $\mathrm{O}$ ideal é selecionar uma faixa de tamanho de sutura entre 4-0 e 6-0 para peixes menores, como os salmonídeos que pesam menos de $200 \mathrm{~g}$, pois este tamanho provavelmente iria manter uma boa tensão de sutura. No entanto, deve-se observar que um fio de sutura 4-0 pode retardar a cura e um fio de sutura 6-0 pode ser tão fino que cortaria o tecido. Daí a importância em fazer um teste antes (Wagner, et al., 2011).

Quando as incisões curaram para minimizar a inflamação que pode ser associada com o material da sutura (Hurty, et al., 2002). A inflamação pode levar à deiscência da ferida, que, no caso de transmissores cirurgicamente colocados, pode levar à perda do transmissor. Para projetos envolvendo peixes que não serão recapturados, a retenção da sutura ao ponto de descamação (não absorção) deve ser antecipada (Harms \& Lewbart, 2000). Retenção de sutura e inflamação associada pode alterar o comportamento dos peixes, o crescimento, o desempenho da natação e a capacidade de sobrevivência (Cannizzo, 2016).

\subsection{Pós-operatório}

Infelizmente existe uma investigação inadequada do manejo da dor pós-operatória em peixes. Embora a percepção da dor por peixes seja assunto para debates, sobre a nocicepção não há dúvidas, e verifica-se que os efeitos comportamentais e fisiológicos da cirurgia em peixes têm o potencial de influenciar os resultados. Além disso, o Animal Welfare Act (única lei federal dos Estados Unidos que regula o tratamento de animais em pesquisas e exposições) não se aplica aos peixes. Portanto, instituições e políticas individuais e requisitos legais que não o Animal Welfare Act (por exemplo: Serviço de Saúde Pública dos EUA e Interagency Research Animal Committee's Principles for the Utilization and Care of Vertebrate Animals 
Used in Testing, Research, and Training) exigem a revisão de projetos envolvendo peixes pela IACUC - Institutional Animal Care and Use Committees (instituto de importância central para a aplicação de leis para pesquisa animal nos Estados Unidos), que por sua vez exige a utilização de sedação adequada, analgesia ou anestesia quando procedimentos que causam mais do que dor leve ou angústia são executados. Porém, a IACUC ainda não têm conhecimento e experiência para avaliar projetos de peixes criteriosamente, especialmente quando os projetos são realizados a campo, como no caso de cirurgias envolvendo a implantação de transmissores. Assim, por falta de conhecimento aprofundado, no momento não é possível fazer recomendações definitivas para uma analgesia apropriada para os peixes no pós-cirúrgico (Harms, 1998; Mulcahy, 2003).

No entanto, o butorfanol a $0,4 \mathrm{mg} / \mathrm{kg}$ intramuscular (IM) em dose única, imediatamente antes da recuperação da anestesia, parece ter bom efeito analgésico (por exemplo, peixes tratados assim nadam na coluna superior de água, são mais ativos e se alimentam mais vigorosamente do que os controles tratados com solução salina). Além disso, não produz efeitos adversos evidentes. O cetoprofeno a $2 \mathrm{mg} / \mathrm{kg} \mathrm{IM}$ em dose única não teve o mesmo efeito, mas reduziu o aumento pós-operatório de creatina quinase, presumivelmente por efeitos anti-inflamatórios. Apesar de uma dose única de analgésico poder não ser o ideal perante uma perspectiva de gestão da dor, é imperativo considerar os efeitos prejudiciais de captura e manipulação de pescado para uma dosagem adicional (Harms, 1998).

\section{Considerações Finais}

Apesar da pouca disponibilidade de material para pesquisa, foi possível com este estudo reunir informações importantes acerca dos procedimentos de cirurgias em peixes. Ficou constatado serem estes animais resistentes a certos procedimentos cirúrgicos, sendo viáveis mais estudos que possam desenvolver novas técnicas que venham a trazer benefícios para a saúde destes animais.

A pesquisa também revelou que são poucos os investimentos nesta área, e que as técnicas utilizadas sofreram poucas melhorias desde que foram desenvolvidas. Apesar disso, algumas inovações têm sido feitas, principalmente no que se refere ao uso de materiais de melhor qualidade para suporte dos peixes durante a cirurgia.

Fica constatado que esta é uma área da medicina veterinária que ainda necessita de expansão, através de pesquisas, melhoria de técnicas e publicações, pois ainda há muitas lacunas a serem preenchidas. 


\section{Referências}

Bizarro, Y. W. S. (2011) Associação do anestésico óleo-de-cravo (eugenol), benzocaína e cloreto de sódio em diferentes concentrações para Tilápia do Nilo submetidos à simulação de transporte. Monografia (Graduação em Medicina Veterinária), Faculdade de Agronomia e Veterinária, Universidade de Brasília, Brasília.

Brattelid, T., Smith, A. J. (2000) Methods of positioning fish for surgery or other procedures out of water. Laboratory Animal, (34), 430-433.

Browser, P. R. (2001) Anesthetic options for fish. In: Gleed, R. D., Ludders, J. W. Recent advances in veterinary anesthesia and analgesia: companion animals. Ithaca, New York, USA: International Veterinary Information Service.

Cannizzo, S. A., Roe S. C., Harms, C. A., \& Stoskopf, M. K. (2016) Effect of water temperature on the hydrolysis of two absorbable sutures used in fish surgery. Facets.

Chambel, J., et al. (2015) The efficacy of MS-222 as anaesthetic agent in four freshwater aquarium fish species. Aquaculture Research.

Cooke, S. J., Graes, B. D. S., Suski, C. D., Ostrand, K. G. (2003) Effects of suture material on incision healing, growth and survival of juvenile largemouth bass implanted with miniature radio transmitters: case study of a novice and experienced fish surgeon. Journal of Fish Biology, (62), 1366-1380.

Coyle, S. D., Durborow, R. M., Tidwell, J. H. (2004) Anesthetics in aquaculture. Southern Regional Aquaculture Center, (3900), 1-6.

Harms, C. (1998) Anesthesia in fish. In: Fowler, M. E., Miller, R. E. Zoo \& wild animal medicine current therapy. (4a ed.), Philadelphia: Saunders.

Harms, C. A., Lewbart, G. A. (2000) Surgery in fish. The Veterinary Clinics of North America. Exotic Animal Practice, 3(3), 759-774. 
Hurty, C. A., Brazik, D. C., Mchugh Law, J., Sakamoto, K., Lewbart, G. A. (2002) Evaluation of the tissue reactions in the skin and body wall of koi (Cyprinus carpio) to five suture materials. Veterinary Record, (151), 324-328.

Koche, J. C. (2011). Fundamentos de metodologia científica. Petrópolis: Vozes. Recuperado de http://www.brunovivas.com/wp-content/uploads/sites/10/2018/07/K\%C3\%B6cheJos\%C3\%A9-Carlos0D0AFundamentos-de-metodologia-cient\%C3\%ADfica-_teoriada0D0Aci\%C3\%AAncia-e-inicia\%C3\%A7\%C3\%A3o-\%C3\%A0-pesquisa.pdf.

Lewbart, G. A., Harms, C. (1999) Building a fish anesthesia delivery system. Exotic DVM, $1.2,25-28$.

Maranho, A. (2007) Peixes ornamentais. In: Cubas, Z. C., Silva, J. C. R., Dias, J. L. C. Tratado de animais selvagens - medicina veterinária. São Paulo: Roca. Cap.6, 44-56.

Mazzafera, P. (2003) Efeito alelopático do extrato alcoólico do cravo-da-índia e eugenol. Revista Brasileira Botânica, 26, 231-238.

Mulcahy, D. M. (2003) Surgical implantation of transmitters into fish. ILAR Journal, 44(4), 295-306.

Neiffer, D. L., Stamper, M. A. (2009) Fish sedation, anesthesia, analgesia, and euthanasia: considerations, methods, and types of drugs. ILAR Journal, 50(4), 343-360.

Pascke, M. S. \& Lanzendorf, F. N. (2017) Diferença entre peixes de água salgada e peixes de água doce. Revista Maiêutica, Indaial, 5(01), 57-68.

Pereira, D. Classificação dos peixes: classes e características. 2015. Recuperado de $<$ http://planetabiologia.com/classificacao-dos-peixes-classe-e-caracteristicas/>.

Pereira-Da-Silva, E. M., Oliveira, R. H. F., Ribeiro, M. A. R., Coppola, M. P. 2009. Efeito anestésico do óleo de cravo em alevinos de lambari. Ciência Rural, 39(6), 1851-1856. 
Pereira, A. S., Shitsuka, D. M., Parreira, F. J., \& Shitsuka, R. (2018). Metodologia da Pesquisa Científica. [e-book]. Ed. UAB/NTE/UFSM. Santa Maria/RS. Recuperado dehttp://repositorio.ufsm.br/bitstream/handle/1/15824/Lic_Computacao_MetodologiaPesquisa Cientifica.pdf?sequence $=1$.

Pier - Pfleger Institute of Environmental Research. Tagging and releasing California coastal fishes. Recuperado de <http://www.pier.org/CA_coastal_tagging.shtml>.

Pires, J. R. (2009) Cirurgia e anestesia em peixes. Rio de Janeiro: UNESA, Qualittas.

Reid, S., Backman, S. (1988) Surgical excision of a tumor in a fish. Canadian Veterinary Journal, 29, 172.

Roubach, R., Gomes, L. C. (2001) O uso de anestésicos durante o manejo de peixes. Panorama da Aquicultura, 11(66), 37-40.

Stoskopf, K. M. (1993) Surgery. In: Fish medicine. Philadelphia: Saunders Company, 7, 9197.

Wagner, G. N., Cooke, S. J. (2005) Methodological approaches and opinions of researchers involved in the surgical implantation of telemetry transmitters in fish. Journal of Aquatic Animal Health, 17, 160-169.

Wagner, G. N., Cooke, S. J., Brown, R. S., Deters, K. A. (2011) Surgical implantation techniques for electronic tags in fish. Reviews in Fish Biology and Fisheries, 21, 71-81.

\section{Porcentagem de contribuição de cada autor no manuscrito}

Jaqueline Nunes Nieri Moreira da Rocha $-40 \%$

Antonia Cleia Bastos - 20\%

Mayra Meneguelli - 10\%

Felipe Mateus Berndt - 10\%

Talita Oliveira Mendonça - 5\%

Dayane da Silva Zanini - 5\%

Igor Mansur Muniz - 10\% 